\title{
On the saddlepoint approximation of the dependence testing bound in memoryless channels
}

\author{
Dadja Anade, Jean-Marie Gorce, Philippe Mary, and Samir M. Perlaza
}

\begin{abstract}
This paper introduces an upper-bound on the absolute difference between: $(a)$ the cumulative distribution function (c.d.f.) of the sum of a finite number of independent and identically distributed (i.i.d) random variables; and $(b)$ a saddlepoint approximation of such c.d.f. This upperbound is general and particularly precise in the regime of large deviations. This result is used to study the dependence testing (DT) bound on the minimum decoding error probability (DEP) in memoryless channels. Within this context, the main results include new lower and upper bounds on the DT bound. As a byproduct, an upper bound on the absolute difference between the exact value of the DT bound and its saddlepoint approximation is obtained. Numerical analysis of these bounds are presented for the case of the binary symmetric channel and the additive white Gaussian noise channel, in which the new bounds are observed to be tight.
\end{abstract}

\section{INTRODUCTION}

This work focuses on providing an upper bound on the minimum decoding error probability (DEP) in point-to-point memoryless channels at a fixed information rate and fixed transmission duration, e.g., $n$ channel uses. More specifically, the objective is to provide tight lower and upper bounds on the dependence testing (DT) bound [1] (Lemma 1 in Section III). The DT bound is known to be difficult to calculate as it involves dealing with the tails of cumulative distribution functions (c.d.f.) of $n$-dimensional random vectors, which justifies seeking for an approximation [1]. This difficulty holds as well for other bounds on the minimum DEP including the meta-converse bound [1] and the random coding union (RCU) bound [1], c.f., [2] and [3]. In the light of this observation, this paper introduces an upper-bound (Theorem 2 in Section IV) on the absolute value of the difference between: $(a)$ the c.d.f. of the sum of a finite number of independent and identically distributed (i.i.d) random variables; and (b) a saddlepoint approximation [4] of such c.d.f. This result is of the same kind of the Berry-Esseen theorem (Theorem 1 in Section IV) that provides an upper bound on the absolute difference between the c.d.f. of the sum of a finite number of i.i.d random variables and the c.d.f. of a Gaussian random variable whose mean and variance are the same as those of the sum [5].

Dadja Anade, Jean-Marie Gorce, and Samir M. Perlaza are with the Laboratoire CITI, a joint laboratory between the Institut National de Recherche en Informatique et en Automatique (INRIA), the Université de Lyon and the Institut National de Sciences Apliquées (INSA) de Lyon. 6 Av. des Arts 69621 Villeurbanne, France. (\{dadja.anade-akpo, jean-marie.gorce, samir.perlaza\}@inria.fr)

Philippe Mary is with the Laboratoire IETR, a joint laboratory between the Centre National de Recherche Scientifique (CNRS), the Universite de Rennes and the Institut National de Sciences Apliquées (INSA) de Rennes. (philippe.mary@insa-rennes.fr)

This work was partially funded by the French National Agency for Research (ANR) under grant ANR-16-CE25-0001.
The Berry-Esseen theorem has played a central role in calculating upper bounds on the DT bound and lower bounds on the meta-converse bound, see for instance, [1] and [6]. These bounds are particularly easy to calculate. Nonetheless, easy computation comes at the expense of loose upper and lower bounds, and thus, uncontrolled approximation errors.

On another note, saddlepoint techniques [4] have been extensively used to approximate existing lower and upper bounds on the minimum DEP. See for instance, [7] and [8] in the case of the RCU bound and the meta-converse bound. Nonetheless, the errors induced by saddlepoint approximations are often neglected due to the fact that calculating them involves a large number of optimizations and numerical integrations.

Within this context, the main results of this paper include new lower and upper bounds on the DT bound. As a byproduct, an upper bound on the absolute difference between the exact value of the DT bound and its saddlepoint approximation is obtained. Numerical analysis of these bounds are presented for the case of the binary symmetric channel (BSC) and the additive white Gaussian noise (AWGN) channel, in which the new bounds are observed to be tight and obtained at low computational cost.

\section{Channel Model}

Consider a point-to-point communication in which a transmitter aims at sending information to one receiver through a noisy memoryless channel. Such a channel can be modeled by a random transformation

$$
\left(\mathcal{X}^{n}, \mathcal{Y}^{n}, P_{\boldsymbol{Y} \mid \boldsymbol{X}}\right)
$$

where $n \in \mathbb{N}$ is the communication duration in channel uses; $\mathcal{X}$ and $\mathcal{Y}$ are respectively the channel input and channel output sets. Given the channel inputs $\boldsymbol{x}=\left(x_{1}, x_{2}, \ldots, x_{n}\right) \in \mathcal{X}^{n}$, the outputs $\boldsymbol{y}=\left(y_{1}, y_{2}, \ldots, y_{n}\right) \in \mathcal{Y}^{n}$ are observed at the receiver with probability:

$$
P_{\boldsymbol{Y} \mid \boldsymbol{X}}(\boldsymbol{y} \mid \boldsymbol{x})=\prod_{t=1}^{n} P_{Y \mid X}\left(y_{t} \mid x_{t}\right)
$$

where, for all $x \in \mathcal{X}, P_{Y \mid X=x} \in \triangle(\mathcal{Y})$, with $\triangle(\mathcal{Y})$ the set of all possible probability distributions whose support is a subset of $\mathcal{Y}$. The objective of the communication is to transmit a message index $i$, which is a realization of a random variable $W$ that is uniformly distributed over the set $\mathcal{W} \triangleq\{1,2, \ldots, M\}$, with $1<M<\infty$. To achieve this objective, the transmitter uses an $(n, M, \lambda)$-code, where $\lambda \in[0,1]$. 
Definition 1 (( $n, M, \lambda)$-code): Given a tuple $(M, n, \lambda) \in$ $\mathbb{N}^{2} \times[0,1]$, an $(n, M, \lambda)$-code for the random transformation in (1) is a system

$$
\{(\boldsymbol{u}(1), \mathcal{D}(1)),(\boldsymbol{u}(2), \mathcal{D}(2)), \ldots,(\boldsymbol{u}(M), \mathcal{D}(M))\}(3)
$$

where for all $(j, \ell) \in \mathcal{W}^{2}$, with $j \neq \ell$ :

$$
\begin{aligned}
& \boldsymbol{u}(j)=\left(u_{1}(j), u_{2}(j), \ldots, u_{n}(j)\right) \in \mathcal{X}^{n}, \\
& \mathcal{D}(j) \cap \mathcal{D}(\ell)=\emptyset, \\
& \bigcup_{j \in \mathcal{W}} \mathcal{D}(j) \subseteq \mathcal{Y}^{n}, \text { and } \\
& \frac{1}{M} \sum_{i=1}^{M} \mathbb{E}_{P_{\boldsymbol{Y} \mid \boldsymbol{X}=\boldsymbol{u}(i)}}\left[\mathbb{1}_{\{Y \notin \mathcal{D}(i)\}}\right] \leqslant \lambda .
\end{aligned}
$$

To transmit message index $i \in \mathcal{W}$, the transmitter uses the codeword $\boldsymbol{u}(i)$. For all $t \in\{1,2, \ldots, n\}$, at channel use $t$, the transmitter inputs the symbol $u_{t}(i)$ into the channel. Assume that at the end of channel use $t$, the receiver observes the output $y_{t}$. After $n$ channel uses, the receiver uses the vector $\boldsymbol{y}=\left(y_{1}, y_{2}, \ldots, y_{n}\right)$ and determines that the symbol $j$ was transmitted if $\boldsymbol{y} \in \mathcal{D}(j)$, with $j \in \mathcal{W}$.

Given the $(n, M, \lambda)$-code described by the system in (3), the DEP of the message index $i$ is $\mathbb{E}_{P_{\boldsymbol{Y} \mid \boldsymbol{X}=\boldsymbol{u}(i)}}\left[\mathbb{1}_{\{Y \notin \mathcal{D}(i)\}}\right]$. As a consequence, the average DEP is

$$
\frac{1}{M} \sum_{i=1}^{M} \mathbb{E}_{P_{\boldsymbol{Y} \mid \boldsymbol{X}=\boldsymbol{u}(i)}}\left[\mathbb{1}_{\{Y \notin \mathcal{D}(i)\}}\right] .
$$

Note that from (4d), the average DEP of such an $(n, M, \lambda)$ code is upper-bounded by $\lambda$. Given a fixed pair $(n, M) \in \mathbb{N}^{2}$, the minimum $\lambda$ for which an $(n, M, \lambda)$-code exists is defined hereunder.

Definition 2: Given a pair $(n, M) \in \mathbb{N}^{2}$, the minimum average DEP for the random transformation in (1), denoted by $\lambda^{*}(n, M)$, is given by

$$
\lambda^{*}(n, M)=\min \{\lambda \in[0,1]: \exists(n, M, \lambda) \text {-code }\} .
$$

When $\lambda$ is chosen accordingly with the reliability constraints, an $(n, M, \lambda)$-code is said to transmit at an information rate $R=\frac{\log _{2}(M)}{n}$ bits per channel use.

\section{The Dependence Testing Bound}

This section describes an upper bound on $\lambda^{*}(n, M)$, for a fixed pair $(n, M) \in \mathbb{N}^{2}$. Given a probability distribution $P_{\boldsymbol{X}} \in \triangle\left(\mathcal{X}^{n}\right)$, let the random variable $\iota(\boldsymbol{X} ; \boldsymbol{Y})$ satisfy

$$
\iota(\boldsymbol{X} ; \boldsymbol{Y}) \triangleq \ln \left(\frac{\mathrm{d} P_{\boldsymbol{X} \boldsymbol{Y}}}{\mathrm{d} P_{\boldsymbol{X}} P_{\boldsymbol{Y}}}(\boldsymbol{X}, \boldsymbol{Y})\right),
$$

where, the function $\frac{\mathrm{d} P_{X Y}}{\mathrm{~d} P_{X} P_{Y}}: \mathcal{X}^{n} \times \mathcal{Y}^{n} \rightarrow \mathbb{R}$ denotes the Radon-Nikodym derivative of the joint probability measure $P_{X Y}$ with respect to the product of probability measures $P_{\boldsymbol{X}} P_{\boldsymbol{Y}}$, with $P_{\boldsymbol{X} Y}=P_{\boldsymbol{X}} P_{\boldsymbol{Y} \mid \boldsymbol{X}}$ and $P_{\boldsymbol{Y}}$ the corresponding marginal. Let the function $T: \mathbb{N}^{2} \times \triangle\left(\mathcal{X}^{n}\right) \rightarrow \mathbb{R}_{+}$be for all $(n, M) \in \mathbb{N}^{2}$ and for all probability distributions $P_{\boldsymbol{X}} \in \triangle\left(\mathcal{X}^{n}\right)$,

$$
\begin{aligned}
T\left(n, M, P_{\boldsymbol{X}}\right)= & \mathbb{E}_{P_{\boldsymbol{X}} P_{\boldsymbol{Y} \mid \boldsymbol{X}}}\left[\mathbb{1}_{\left\{\iota(\boldsymbol{X} ; \boldsymbol{Y}) \leqslant \ln \left(\frac{M-1}{2}\right)\right\}}\right] \\
& +\frac{M-1}{2} \mathbb{E}_{P_{\boldsymbol{X}} P_{\boldsymbol{Y}}}\left[\mathbb{1}_{\left\{\iota(\boldsymbol{X} ; \boldsymbol{Y})>\ln \left(\frac{M-1}{2}\right)\right\}}\right] .
\end{aligned}
$$

Using this notation, the following lemma describes the dependence testing bound.

Lemma 1 (Dependence testing bound [1]): Given a pair $(n, M) \in \mathbb{N}^{2}$, the following holds for all $P_{\boldsymbol{X}} \in \triangle\left(\mathcal{X}^{n}\right)$, with respect to the random transformation in (1):

$$
\lambda^{*}(n, M) \leqslant T\left(n, M, P_{\boldsymbol{X}}\right),
$$

with the function $T$ defined in (7).

Note that the input probability distribution $P_{\boldsymbol{X}}$ in Lemma 1 can be chosen among all possible probability distributions $P_{\boldsymbol{X}} \in \triangle\left(\mathcal{X}^{n}\right)$ to minimize the right-hand side of (8), which improves the bound. Note also that with some lost of optimality, the optimization domain can be constrained to the set of probability distributions for which for all $\boldsymbol{x} \in \mathcal{X}^{n}$,

$$
P_{\boldsymbol{X}}(\boldsymbol{x})=\prod_{t=1}^{n} P_{X}\left(x_{t}\right)
$$

with $P_{X} \in \triangle(\mathcal{X})$. Hence, subject to (2), the random variable $\iota(\boldsymbol{X} ; \boldsymbol{Y})$ in $(8)$ can be written as the sum of the i.i.d. random variables, i.e.,

$$
\iota(\boldsymbol{X} ; \boldsymbol{Y})=\sum_{t=1}^{n} \iota\left(X_{t} ; Y_{t}\right) .
$$

This observation motivates the study of the c.d.f. of random variables consisting of the sum of a finite number of i.i.d. random variables.

\section{SumS OF INDEPENDENT AND IDENTICALLY DISTRIBUTED RANDOM VARIABLES}

Let $Y$ be a real-valued random variable with probability distribution $P_{Y}$ and moment generating function $\varphi_{Y}$. Let the functions $\mu_{Y}: \mathbb{R} \rightarrow \mathbb{R}, V_{Y}: \mathbb{R} \rightarrow \mathbb{R}$, and $\xi_{Y}: \mathbb{R} \rightarrow \mathbb{R}$ be such that for all $t \in \mathbb{R}$,

$$
\begin{aligned}
& \mu_{Y}(t) \triangleq \mathbb{E}_{P_{Y}}\left[\frac{Y \exp (t Y)}{\varphi_{Y}(t)}\right], \\
& V_{Y}(t) \triangleq \mathbb{E}_{P_{Y}}\left[\frac{\left|Y-\mu_{Y}(t)\right|^{2} \exp (t Y)}{\varphi_{Y}(t)}\right], \text { and } \\
& \xi_{Y}(t) \triangleq \mathbb{E}_{P_{Y}}\left[\frac{\left|Y-\mu_{Y}(t)\right|^{3} \exp (t Y)}{\varphi_{Y}(t)}\right] .
\end{aligned}
$$

Let also the function $\zeta_{Y}: \mathbb{R}^{2} \times \mathbb{N} \rightarrow \mathbb{R}$ be defined such that for all $(\theta, a, n) \in \mathbb{R}^{2} \times \mathbb{N}$,

$\zeta_{Y}(\theta, a, n)=\mathbb{1}_{\{\theta>0\}}+$

$(-1)^{\mathbb{1}_{\{\theta>0\}}} \exp \left(\frac{1}{2} n \theta^{2} V_{Y}(\theta)+n \ln \left(\varphi_{Y}(\theta)\right)-\theta a\right) Q\left(|\theta| \sqrt{n V_{Y}(\theta)}\right)$,

where $Q: \mathbb{R} \rightarrow[0,1]$ is the complementary c.d.f. of the standard Gaussian distribution. The following theorem, known as the Berry-Esseen theorem [5], introduces an upper-bound 
on the absolute value of the difference between: $(a)$ the c.d.f. of the sum of a finite number of i.i.d. random variables; and (b) the c.d.f. of a particular Gaussian random variable.

Theorem 1 (Berry-Esseen [5]): Let $Y_{1}, Y_{2}, \ldots, Y_{n}$ be i.i.d random variables with probability distribution $P_{Y}$. Let also $Z_{n}$ be a Gaussian random variable with mean $n \mu_{Y}(0)$, variance $n V_{Y}(0)$ and c.d.f. denoted by $F_{Z_{n}}$. Then, the c.d.f. of the random variable $X_{n}=Y_{1}+Y_{2}+\ldots+Y_{n}$, denoted by $F_{X_{n}}$, satisfies

$$
\sup _{a \in \mathbb{R}}\left|F_{X_{n}}(a)-F_{Z_{n}}(a)\right| \leqslant \min \left(1, \frac{c \xi_{Y}(0)}{\sqrt{n\left(V_{Y}(0)\right)^{3}}}\right),
$$

where $c=0.476$ and the functions $\mu_{Y}, V_{Y}$ and $\xi_{Y}$ are defined in (11), (12) and (13).

The choice of $c=0.476$ in Theorem 1 is justified in [9]. Intuitively, Theorem 1 states that for all $a \in \mathbb{R}$, the value of $F_{X_{n}}(a)$ can be "approximated" by the value $F_{Z_{n}}(a)$ up to an "approximation error" of at most $\frac{c \xi_{Y}(0)}{\sqrt{n\left(V_{Y}(0)\right)^{3}}}$. From this perspective, the main drawback of Theorem 1 is that the upper bound on the "approximation error" $\left|F_{X_{n}}(a)-F_{Z_{n}}(a)\right|$ does not depend on the exact value of $a$. More importantly, for some values of $a$ and $n$, the right-hand side of (15), i.e., the bound on the "approximation error" resulting from Theorem 1 , might be particularly big, which leads to irrelevant results. The following theorem attempts to overcome this drawback.

Theorem 2: Let $Y_{1}, Y_{2}, \ldots, Y_{n}$ be i.i.d. random variables with probability distribution $P_{Y}$ and moment generating function $\varphi_{Y}$. Let also $F_{X_{n}}$ be the c.d.f. of the random variable $X_{n}=Y_{1}+Y_{2}+\ldots+Y_{n}$. Hence, for all $a \in \operatorname{int} \mathcal{C}_{X_{n}}$, with $\mathcal{C}_{X_{n}}$ denoting the convex hull of $\operatorname{supp} P_{X_{n}}$ and $\operatorname{int} \mathcal{C}_{X_{n}}$ the interior of $\mathcal{C}_{X_{n}}$, it holds that

$$
\begin{aligned}
& \left|F_{X_{n}}(a)-\zeta_{Y}\left(\theta^{\star}, a, n\right)\right| \\
& \leqslant \exp \left(n \ln \left(\varphi_{Y}\left(\theta^{\star}\right)\right)-\theta^{\star} a\right) \min \left(1, \frac{2 c \xi_{Y}\left(\theta^{\star}\right)}{\left(V_{Y}\left(\theta^{\star}\right)\right)^{3 / 2} \sqrt{n}}\right),
\end{aligned}
$$

where $c=0.476, \theta^{\star}$ is the unique solution in $\theta$ to

$$
n \mu_{Y}(\theta)=a,
$$

and the functions $\mu_{Y}, V_{Y}, \xi_{Y}$, and $\zeta_{Y}$ are defined in (11), (12), (13) and (14), respectively.

Proof: The proof of Theorem 2 is presented in [10]. The relevance of Theorem 2 is that given a pair $(a, n) \in \mathbb{R} \times \mathbb{N}$, the value $F_{X_{n}}(a)$ can be "approximated" by $\zeta_{Y}\left(\theta^{\star}, a, n\right)$ up to an "approximation error" that is not bigger than $\exp \left(n \ln \left(\varphi_{Y}\left(\theta^{\star}\right)\right)-\theta^{\star} a\right) \min \left(1, \frac{2 c \xi_{Y}\left(\theta^{\star}\right)}{\left(V_{Y}\left(\theta^{\star}\right)\right)^{3 / 2} \sqrt{n}}\right)$. Note that this upper-bound depends not only on $a$ but also on $\theta^{\star}$, which is chosen to satisfy (17). The motivation of this choice is thoroughly discussed in [10].

Note that in [4], the function $\zeta_{Y}$ in (14) is referred to as the saddlepoint approximation of $F_{X_{n}}$ in (16). Nonetheless, from the results in [4], it is often difficult to study the "approximation error".

An interesting observation from Theorem 2 is that the maximum of $\exp \left(n \ln \left(\varphi_{Y}\left(\theta^{\star}\right)\right)-\theta^{\star} a\right)$ is one and it is reached when $a=n \mathbb{E}_{P_{Y}}[Y]=\mathbb{E}_{P_{X_{n}}}\left[X_{n}\right]$. In this case, $\theta^{\star}=0$, and thus, the "approximation error" obtained from Theorem 2 is bigger than the one obtained using Theorem 1. In a nutshell, for values of $a$ in the vicinity of $n \mathbb{E}_{P_{Y}}[Y]=\mathbb{E}_{P_{X_{n}}}\left[X_{n}\right]$, it is more interesting to use Theorem 1 instead of Theorem 2.

Alternatively, given that $n \ln \left(\varphi_{Y}\left(\theta^{\star}\right)\right)-\theta^{\star} a$ is a non-positive and concave function of $a$, when $\left|a-n \mathbb{E}_{P_{Y}}[Y]\right|=\mid a-$ $\mathbb{E}_{P_{X_{n}}}\left[X_{n}\right] \mid>\gamma$, with $\gamma$ sufficiently large, it follows that

$$
\exp \left(n \ln \left(\varphi_{Y}\left(\theta^{\star}\right)\right)-\theta^{\star} a\right)<\min \left(1, \frac{c \xi_{Y}(0)}{\sqrt{n\left(V_{Y}(0)\right)^{3}}}\right) .
$$

Hence, in this case, the right-hand side of (16) is always smaller than the right-hand side of (15). This implies that in the regime of large deviations, it is more interesting to use Theorem 2 instead of Theorem 1.

\section{On the Calculation of the Dependence Testing BOUND}

This section focuses on providing upper and lower bounds on the function $T$ in (7), for some given values $(n, M) \in \mathbb{N}^{2}$ and a given distribution $P_{\boldsymbol{X}} \in \triangle\left(\mathcal{X}^{n}\right)$. These bounds become significantly relevant when the exact value of $T\left(n, M, P_{\boldsymbol{X}}\right)$ cannot be calculated with respect to the random transformation in (1). In such a case, providing upper and lower bounds on $T\left(n, M, P_{\boldsymbol{X}}\right)$ helps in approximating its exact value subject to an error sufficiently small such that the approximation is relevant.

\section{A. Existing Results}

For all $P_{X} \in \triangle(\mathcal{X})$, let $\mu\left(P_{X}\right) \triangleq \mathbb{E}_{P_{X} P_{Y \mid X}}[\iota(X ; Y)]$ denote the first moment; $\sigma\left(P_{X}\right) \triangleq \mathbb{E}_{P_{X} P_{Y \mid X}}[(\iota(X ; Y)-$ $\left.\left.\mu\left(P_{X}\right)\right)^{2}\right]$ denote the second central moment; and $\xi\left(P_{X}\right) \triangleq$ $\mathbb{E}_{P_{X} P_{Y \mid X}}\left[\left|\iota(X ; Y)-\mu\left(P_{X}\right)\right|^{3}\right]$ denote the third absolute central moment of the random variable $\iota(X ; Y)$. Using this notation, consider the functions $D: \mathbb{N}^{2} \times \triangle(\mathcal{X}) \rightarrow \mathbb{R}_{+}$and $N: \mathbb{N}^{2} \times \triangle(\mathcal{X}) \rightarrow \mathbb{R}_{+}$such that for all $(n, M) \in \mathbb{N}^{2}$ and for all $P_{X} \in \triangle(\mathcal{X})$,

$D\left(n, M, P_{X}\right)=\max \left(0, \alpha\left(n, M, P_{X}\right)-\frac{c \xi\left(P_{X}\right)}{\sigma\left(P_{X}\right)^{\frac{3}{2}} \sqrt{n}}\right)$ and(19)

$N\left(n, M, P_{X}\right)=$

$\min \left(1, \alpha\left(n, M, P_{X}\right)+\frac{3 c \xi\left(P_{X}\right)}{\sigma\left(P_{X}\right)^{\frac{3}{2}} \sqrt{n}}+\frac{2 \ln (2)}{\sigma\left(P_{X}\right)^{\frac{1}{2}} \sqrt{2 n \pi}}\right)$,

where, $c=0.476$ and

$$
\alpha\left(n, M, P_{X}\right) \triangleq Q\left(\frac{n \mu\left(P_{X}\right)-\ln \left(\frac{M-1}{2}\right)}{\sqrt{n \sigma\left(P_{X}\right)}}\right) .
$$

Using this notation, the following theorem introduces a lower bound and an upper bound on $T$ in (7).

Theorem 3: Given a pair $(n, M) \in \mathbb{N}^{2}$, for all input distributions $P_{\boldsymbol{X}} \in \triangle\left(\mathcal{X}^{n}\right)$ subject to (9), the following holds with respect to the random transformation in (1),

$$
D\left(n, M, P_{X}\right) \leqslant T\left(n, M, P_{X}\right) \leqslant N\left(n, M, P_{X}\right),
$$

where the functions $T, D$ and $N$ are defined in (7), (19) and (20), respectively. 
The proof of Theorem 3 is presented in [2]. Essentially, it consists in using Theorem 1 for independently upper and lower bounding the terms $\mathbb{E}_{P_{\boldsymbol{X}} P_{\boldsymbol{Y} \mid \boldsymbol{X}}}\left[\mathbb{1}_{\left\{\iota(\boldsymbol{X} ; \boldsymbol{Y}) \leqslant \ln \left(\frac{M-1}{2}\right)\right\}}\right]$ and $\mathbb{E}_{P_{\boldsymbol{X}} P_{\boldsymbol{Y}}}\left[\mathbb{1}_{\left\{\iota(\boldsymbol{X} ; \boldsymbol{Y})>\ln \left(\frac{M-1}{2}\right)\right\}}\right]$ in (7). In [2], the function $\alpha\left(n, M, P_{X}\right)$ in (21) is often referred to as an "approximation" of $T\left(n, M, P_{X}\right)$. Nonetheless, as shown in the following section, the "approximation error" is such that it often leads to irrelevant results for small values of $n$.

\section{B. New Results}

Given a distribution $P_{X} \in \triangle(\mathcal{X})$, the moment generating function of $\iota(X ; Y)$ is

$$
\varphi\left(P_{X}, \theta\right) \triangleq \mathbb{E}_{P_{X} P_{Y \mid X}}[\exp (\theta \iota(X ; Y))],
$$

with $\theta \in \mathbb{R}$. For all $P_{X} \in \triangle(\mathcal{X})$ and for all $\theta \in \mathbb{R}$, consider the following functions:

$\mu\left(P_{X}, \theta\right) \triangleq \mathbb{E}_{P_{X} P_{Y \mid X}}\left[\frac{\iota(X ; Y) \exp (\theta \iota(X ; Y))}{\varphi\left(P_{X}, \theta\right)}\right]$,
$V\left(P_{X}, \theta\right) \triangleq \mathbb{E}_{P_{X} P_{Y \mid X}}\left[\left|\iota(X ; Y)-\mu\left(P_{X}, \theta\right)\right|^{2} \frac{\exp (\theta \iota(X ; Y))}{\varphi\left(P_{X}, \theta\right)}\right]$,

and

$\xi\left(P_{X}, \theta\right) \triangleq \mathbb{E}_{P_{X} P_{Y \mid X}}\left[\left|\iota(X ; Y)-\mu\left(P_{X}, \theta\right)\right|^{3} \frac{\exp (\theta \iota(X ; Y))}{\varphi\left(P_{X}, \theta\right)}\right]$.

Using this notation consider the functions $\beta_{1}: \mathbb{N}^{2} \times \mathbb{R} \times$ $\triangle(\mathcal{X}) \rightarrow \mathbb{R}_{+}$and $\beta_{2}: \mathbb{N}^{2} \times \mathbb{R} \times \triangle(\mathcal{X}) \rightarrow \mathbb{R}_{+}$respectively defined in (26) and (27) in the top of next page. Consider also the following functions:

$$
\begin{aligned}
& G_{1}\left(n, M, \theta, P_{X}\right)=\beta_{1}\left(n, M, \theta, P_{X}\right) \\
& -\frac{2 c \xi\left(P_{X}, \theta\right)}{V\left(P_{X}, \theta\right)^{3 / 2} \sqrt{n}} \exp \left(n \ln \left(\varphi\left(P_{X}, \theta\right)\right)-\theta \ln \left(\frac{M-1}{2}\right)\right), \\
& G_{2}\left(n, M, \theta, P_{X}\right)=\beta_{2}\left(n, M, \theta, P_{X}\right) \\
& -\frac{2 c \xi\left(P_{X}, \theta\right)}{V\left(P_{X}, \theta\right)^{3 / 2} \sqrt{n}} \exp \left(n \ln \left(\varphi\left(P_{X}, \theta\right)\right)-(\theta+1) \ln \left(\frac{M-1}{2}\right)\right)
\end{aligned}
$$

and

$G\left(n, M, \theta, P_{X}\right)=\max \left(0, G_{1}\left(n, M, \theta, P_{X}\right)\right)$

$+\frac{M-1}{2} \max \left(0, G_{2}\left(n, M, \theta, P_{X}\right)\right)$.

The following theorem introduces a new lower bound and a new upper bound on $T$ in (7).

Theorem 4: Given a pair $(n, M) \in \mathbb{N}^{2}$, for all input distributions $P_{\boldsymbol{X}} \in \triangle\left(\mathcal{X}^{n}\right)$ subject to (9), the following holds with respect to the random transformation in (1),

$$
G\left(n, M, \theta, P_{X}\right) \leqslant T\left(n, M, P_{X}\right) \leqslant S\left(n, M, \theta, P_{X}\right)
$$

where, $\theta$ is the unique solution in $t$ to

$$
n \mu\left(P_{X}, t\right)=\ln \left(\frac{M-1}{2}\right),
$$

and the functions T, $G$ and $S$ are defined in (7), (24) and (25), with $c=0.476$.
Proof: The proof of Theorem 4 is presented in [10]. In a nutshell, the proof consists in using Theorem 2 for independently bounding the terms $\mathbb{E}_{P_{\boldsymbol{X}} P_{\boldsymbol{Y} \mid \boldsymbol{X}}}\left[\mathbb{1}_{\left\{\iota(\boldsymbol{X} ; \boldsymbol{Y}) \leqslant \ln \left(\frac{M-1}{2}\right)\right\}}\right]$ and $\mathbb{E}_{P_{\boldsymbol{X}} P_{\boldsymbol{Y}}}\left[\mathbb{1}_{\left\{\iota(\boldsymbol{X} ; \boldsymbol{Y}) \geqslant \ln \left(\frac{M-1}{2}\right)\right\}}\right]$ in (8).

The function

$$
\beta\left(n, M, \theta, P_{X}\right)=\beta_{1}\left(n, M, \theta, P_{X}\right)+\frac{M-1}{2} \beta_{2}\left(n, M, \theta, P_{X}\right),
$$

with $\beta_{1}$ in (26) and $\beta_{2}$ in (27), is referred to as a saddlepoint approximation of the function $T$ in (7).

In the following section, the approximations on the function $T$ in (7) obtained by using both Theorem 3 and Theorem 4 are compared in the context of two canonical communications channels: the BSC and the AWGN channel.

\section{EXAMPLES}

\section{A. Binary Symmetric Channel}

Consider a BSC with cross-over probability $\delta=0.11$. The focus is on the analysis of the upper bound on the DEP (Definition 2) at an information rate $R=0.32$ bits per channel use. In Figure 1, the function $T$ in (7) is approximated by using Theorem 3 and Theorem 4. The approximation obtained by using the former, i.e., $\alpha\left(n, 2^{n R}, P_{X}\right)$ in (21), is plotted in black diamonds, whereas the corresponding lower and upper bounds, i.e., $D\left(n, 2^{n R}, P_{X}\right)$ in (19) and $N\left(n, 2^{n R}, P_{X}\right)$ in (20), are respectively plotted in red circles and blue squares. On the other hand, the approximation obtained by using the new result, i.e., $\beta\left(n, 2^{n R}, \theta, P_{X}\right)$ in (30), is plotted in black stars, whereas the corresponding upper and lower bounds, i.e., $S\left(n, 2^{n R}, \theta, P_{X}\right)$ in (25) and $G\left(n, 2^{n R}, \theta, P_{X}\right)$ in (24), are plotted in blue upward-pointing triangles and red downwardpointing triangles. In all cases, $P_{X}$ is the uniform distribution and $\theta$ is chosen to be the unique solution in $t$ of the equality in (29).

\section{B. Additive White Gaussian Noise Channel}

Consider a real-valued AWGN channel with discrete channel inputs, $\mathcal{X}=\{-1,1\}$, and signal to noise ratio $\mathrm{SNR}=1$. The focus is on the analysis of the upper bound on the DEP (Definition 2) at an information rate $R=0.39$ bits per channel use. In Figure 2, the function $T$ in (7) is approximated by using Theorem 3 and Theorem 4 . In all cases, $P_{X}$ is the uniform distribution and $\theta$ is chosen to be the unique solution in $t$ of the equality in (29). The plots follow the same code of colors and markers as in Figure 1.

\section{Discussion}

Let $\theta$ be the unique solution in $t$ to (29). Note that the approximation of the function $T$ in (7) by the function $\alpha$ in (21) might lead to erroneous conclusions. For instance, the exact value of $T\left(n, M, P_{X}\right)$ is between $S\left(n, M, \theta, P_{X}\right)$ and $G\left(n, M, \theta, P_{X}\right)$ (Theorem 4), nonetheless, in the case of the BSC in Figure 1, when $n>1000$ channel uses, it holds that $\alpha\left(n, M, P_{X}\right)<G\left(n, M, \theta, P_{X}\right)$. In this case, approximating $T$ by $\alpha$ is too optimistic. Note also that the lower bound $D\left(n, M, P_{X}\right)$ obtained from Theorem 3 is nonpositive in this case, and thus, does not appear in Figure 1 and Figure 2. On the other hand, the upper bound $N\left(n, M, P_{X}\right)$ is 


$$
\begin{aligned}
& S\left(n, M, \theta, P_{X}\right)=\min \left(1, \beta\left(n, M, \theta, P_{X}\right)+\frac{4 c \xi\left(P_{X}, \theta\right)}{\left(V\left(P_{X}, \theta\right)\right)^{3 / 2} \sqrt{n}} \exp \left(n \ln \left(\varphi\left(P_{X}, \theta\right)\right)-\theta \ln \left(\frac{M-1}{2}\right)\right)\right) \\
& \beta_{1}\left(n, M, \theta, P_{X}\right)=\mathbb{1}_{\{\theta>0\}}+(-1)^{\mathbb{1}_{\{\theta>0\}}} \exp \left(n \ln \left(\varphi\left(P_{X}, \theta\right)\right)-\theta \ln \left(\frac{M-1}{2}\right)+\frac{1}{2} \theta^{2} n V\left(P_{X}, \theta\right)\right) Q\left(\sqrt{n V\left(P_{X}, \theta\right)}|\theta|\right) \cdot(26) \\
& \beta_{2}\left(n, M, \theta, P_{X}\right)=\mathbb{1}_{\{\theta \leqslant-1\}}+(-1)^{\mathbb{1}_{\{\theta \leqslant-1\}}} \exp \left(n \ln \left(\varphi\left(P_{X}, \theta\right)\right)-(\theta+1) \ln \left(\frac{M-1}{2}\right)+\frac{1}{2}(\theta+1)^{2} n V\left(P_{X}, \theta\right)\right) Q\left(\sqrt{n V\left(P_{X}, \theta\right)}|\theta+1|\right) \cdot(27)
\end{aligned}
$$

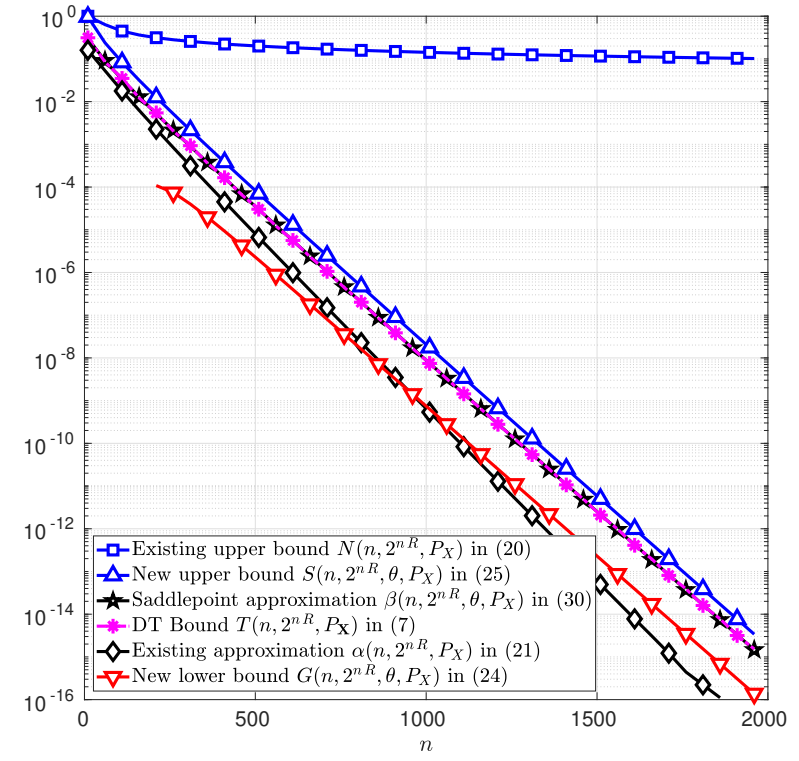

Fig. 1: Approximation of the function $T$ in (7) as a function of the blocklength $n$ for the case of a binary symmetric channel with cross-over probability $\delta=0.11$ at information rate $R=$ 0.32 bits per channel use, $P_{X}$ the uniform distribution and $\theta$ chosen to be the unique solution in $t$ of the equality in (29).

several orders of magnitude far away from the approximation $\alpha\left(n, M, P_{X}\right)$. From this perspective, a proper analysis on the dependence testing bound (Lemma 1) based on Theorem 3 does not lead to relevant conclusions.

Finally, note that in the case of the BSC, the function $T$ in (7) can be calculated exactly and thus, it is plotted in Figure 1 in magenta asterisks. Therein, it can be observed that both the saddlepoint approximation $\beta$ and the function $T$ overlap. These observations are in line with those reported in [7], in which the saddlepoint approximation of the RCU bound and the meta-converse bound are both shown to be precise approximations.

\section{REFERENCES}

[1] Y. Polyanskiy, H. V. Poor, and S. Verdú, "Channel coding rate in the finite blocklength regime," IEEE Transactions on Information Theory, vol. 56, no. 5, pp. 2307-2359, May 2010.

[2] E. MolavianJazi, "A unified approach to Gaussian channels with finite blocklength,” Ph.D. dissertation, University of Notre Dame, Jul. 2014.

[3] T. Erseghe, "Coding in the finite-blocklength regime: Bounds based on Laplace integrals and their asymptotic approximations," IEEE Transactions on Information Theory, vol. 62, no. 12, pp. 6854-6883, Dec. 2016.

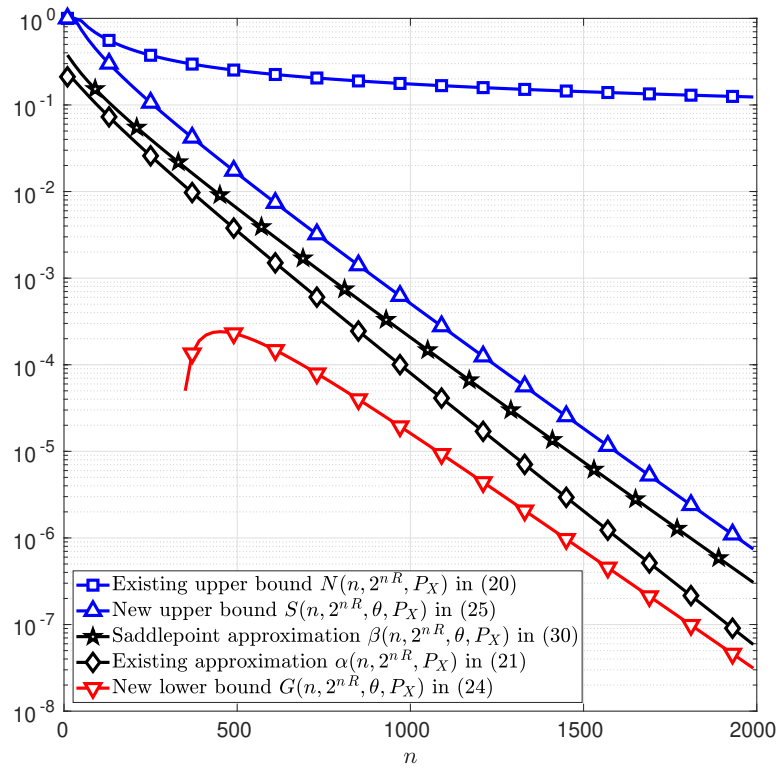

Fig. 2: Approximation of the function $T$ in (7) as a function of the blocklength $n$ for the case of a real-valued AWGN channel with discrete channel inputs, $\mathcal{X}=\{-1,1\}$, and signal to noise ratio $\mathrm{SNR}=1$ at information rate $R=0.39$ bits per channel use, $P_{X}$ the uniform distribution and $\theta$ chosen to be the unique solution in $t$ of the equality in (29).

[4] J. L. Jensen, Saddlepoint Approximations. New York, NY, USA: Clarendon press - Oxford, 1995.

[5] W. Feller, An Introduction to Probability Theory and Its Applications, 2nd ed. New York, NY, USA: John Wiley and Sons, 1971, vol. 2.

[6] E. MolavianJazi and J. N. Laneman, "A second-order achievable rate region for Gaussian multi-access channels via a central limit theorem for functions," IEEE Transactions on Information Theory, vol. 61, no. 12, pp. 6719-6733, Dec. 2015.

[7] J. Font-Segura, G. Vazquez-Vilar, A. Martinez, A. G. i Fàbregas, and A. Lancho, "Saddlepoint approximations of lower and upper bounds to the error probability in channel coding," in Proc. of the 52nd Annual Conference on Information Sciences and Systems (CISS), Princeton, NJ, USA, Mar. 2018, pp. 1-6.

[8] A. Martinez, J. Scarlett, M. Dalai, and A. G. i Fàbregas, "A complexintegration approach to the saddlepoint approximation for randomcoding bounds," in Proc. of the 11th International Symposium on Wireless Communications Systems (ISWCS), Barcelona, Spain, Aug. 2014, pp. 618-621.

[9] I. Shevtsova, "On the absolute constants in the Berry-Esseen type inequalities for identically distributed summands," ArXiv e-prints, Nov. 2011.

[10] D. Anade, J.-M. Gorce, P. Mary, and S. M. Perlaza, "An upper bound on the error induced by saddlepoint approximations - Applications to information theory," INRIA Grenoble - Rhône-Alpes, Tech. Rep. 9329, Feb. 2020. 